\title{
MODELO DE VALORACIÓN DE LA GESTIÓN PRODUCTIVA DE LA PIÑA, PARA SU CERTIFICACIÓN
}

\author{
FEDERICO LI BONILLA \\ Escuela de Ciencias de la Administración \\ Universidad Estatal a Distancia, Costa Rica \\ fli@uned.ac.cr
}

\section{RESUMEN}

Considerando la importancia de la producción de piña en la economía costarricense, se propone un modelo de medición cualitativa y cuantitativa de la calidad en la operación agroindustrial de la piña, para la certificación del proceso del cultivo, recolecta, clasificación de la fruta y embalaje. Dicho modelo, además de que permite cuantificar el rendimiento de las diferentes fases de esta agroindustria, puede servir a los productores y empacadoras como instrumento para presentar ante la Unión Europea, cuando esta viene a certificar los procesos de siembra y recolección en las fincas, así como los procesos de las empacadoras de piña. Asimismo, permite tener controles que ayuden a maximizar su eficiencia y eficacia.

PALABRAS CLAVE: MODELO, PIÑA, AGROINDUSTRIA, CERTIFICACIÓN.

\section{ABSTRACT}

Considering the importance of pineapple production for Costa Rica's economy, this article proposes a qualitative and quantitative model to determine the quality of the pineapple agribusiness operation and to certify the cultivation, harvest, classification and packing processes of the fruit. This model, besides quantifying the performance of the different phases of this agricultural industry, can also help producers and packers to meet the terms of the European Union when it comes to certify the sowing and harvest processes of farms and pineapple canneries. It also helps maximize their efficiency and effectiveness.

KEYWORDS: MODEL, PINEAPPLE, AGROINDUSTRY, CERTIFICATION

\section{INTRODUCCIÓN}

Aunque ciertamente desde principios de este siglo XXI Costa Rica ha estado inmersa en la economía terciaria, muy enfatizada en los servicios y el conocimiento, es cierto también que desde 1980 ha estado involucrada en el desarrollo de la producción de piña. En el contexto de esta sociedad de consumo, la estrategia que Costa Rica empezó a utilizar ha consistido en establecer la diferenciación de las variedades de piña que produce, lo cual se ve reflejado en el hecho de que el país es líder en la venta de piña en fruta y piña orgánica, y está empezando a incursionar en la venta de jugo de piña como una nueva estrategia de mercadeo.

La producción de piña está concentrada en grandes empresas como PINDECO, Del Monte, FYFFES, CHIQUITA y DOLE, así como cientos de pequeños parceleros. Por su parte, la Cámara Nacional de Productores y Exportadores de Piña (CANAPEP) estima que actualmente hay en el país alrededor de 1300 productores de piña. Los pequeños están ubicados especialmente en las zonas norte y Caribe, donde se presentó una gran expansión durante los últimos años. También se cultiva piña en la zona sur.

Pero los países desarrollados que consumen la piña, son mercados maduros y exigentes. Es así como con regularidad la producción de piña de cualquier país del mundo que desee exportar a Europa, es sometida a una revisión y certificación de su manejo; para poder ser importada desde Europa. Es por eso que se propone este modelo de valora- 
ción de gestión de la producción de piña, como un modelo empírico que pueda dar soporte a la certificación de la agroindustria piñera.

\section{Las frutas tropicales}

En el mundo existen alrededor de 500 especies frutales, que crecen en diferentes climas como: tropical, subtropical y templado. Pese a la cantidad de variedades de frutos que hay en nuestro planeta, únicamente alrededor de 15 frutas se explotan a escala comercial.

Se considera que aproximadamente 180 millones de toneladas métricas (tm), son producidas en la zona tropical; siendo 360 millones de tm la producción mundial. Los mayores productores de fruta a nivel mundial son Brasil, India, Estados Unidos y China (Llanos, 1998; INFOAGRO'; Acuña, 2006; PROCOMER, 2010)

En los últimos años se ha venido dando un incremento en la ingesta de frutas tropicales, ya sean transformadas o en presentación fresca, especialmente en los países desarrollados.

El incremento en el consumo de frutas se debe en parte a la expansión geográfica de los productos, y está basado en un comercio cada vez más rápido, seguro y económico. Aunado a esto, está el incremento de la actividad turística, así como el progreso de las nuevas técnicas e innovaciones en la transformación y empaquetado de las frutas (Llanos, 1998).

Además, debe considerarse que la sociedad del siglo XXI, está experimentando una concepción particular del elemento tiempo, el cual es limitado; por ende, el tiempo que se destina a la preparación de los alimentos es cada vez menor. En ese contexto, las frutas tropicales aparecen como una opción interesante, ya que son rápidas de preparar, y además constituyen un excelente complemento nutricional.

1. Sistema de Información del Sector Agropecuario Costarricense, del Ministerio de Agricultura y Ganadería (MAG).
Según Fernández (2000) el consumo de frutas frescas en la Unión Europea (UE) es de aproximadamente 30 millones de tm anuales, lo cual equivale a 81 kilos de consumo per cápita. Es importante anotar que se producen diferencias de consumo entre los diferentes países que conforman la Unión Europea; así por ejemplo, el consumo per cápita/año de Grecia es de 155 kilos y el de Alemania es de 112 kilos, cifras que los convierten en los mayores consumidores de frutas de Europa. Seguido por Austria con 96 kilos; Italia con 91 kilos; y España con un consumo per cápita/año de 83 kilos.

\section{Las frutas tropicales en la Unión Europea}

En la Comunidad Económica Europea la comercialización de frutas tropicales es realizada principalmente por las grandes cadenas de distribución, empresas sumamente organizadas que cuentan con enormes almacenes y medios óptimos de distribución para llegar con prontitud a los detallistas. Dichas cadenas convierten esta actividad en un proceso sumamente competitivo y poderoso, lo cual les permite ejercer presión para obtener la mejor calidad, la mejor presentación y los precios más atractivos.

A continuación se expondrá, en orden de importancia, las frutas tropicales que tienen mayor demanda de consumo en la Unión Europea². La participación de la piña en el mercado europeo es de un 40,8 \%, seguida por los melones con $22,8 \%$, los aguacates con $15,9 \%$, y finalmente las guayabas, mangos y mangostanes con 15,5\% (PROCOMER, 2010).

\section{La piña}

La piña es endémica de la zona brasilera del Mattogroso y fue diseminada en el Caribe por los españoles. La piña no se conoce en estado verdaderamente silvestre y no parece derivarse

2. Exportadas a la Unión Europea por todos los países del planeta. 
de las otras especies de frutos comestibles del género Ananas de la familia bromelieaceae, tales como A. bracteatus, A. fritzmuelleri, A. erectifolia y A. ananasioides, las cuales producen frutos pequeños, casi sin semillas (Collins, 1949; 1960).

Los continentes con mayor participación en el cultivo de la piña son Asia, con una participación del 53\%, América con el 29,6\% y con el 16,3\% África. El mayor productor del mundo es Tailandia (PROCOMER, 2010)

El mercado internacional se comporta positivamente para productos como la piña, dándose una demanda importante por la fruta fresca de este tipo, que no solo sigue en auge, sino que es una de las más cotizadas a nivel internacional: la piña fresca de Costa Rica.

El crecimiento en el cultivo de la piña en Costa Rica ha sido vertiginoso desde 1980, pues pasó de tener sembrado solo 500 hectáreas, a tener un aproximado de 51000 hectáreas cultivadas en 2009.

En el mercado de la UE se ofrecen diferentes variedades de piñas, siendo las más solicitadas la Cayenne Lisse y la Annanas Bouteille.

Los principales exportadores de piña a Europa se pueden apreciar en la tabla 1.; se da una peculiaridad en la calidad de la piña producida en Costa Rica siendo la de mejor cotización.

TABLA 1

\section{Principales exportadores de piña a Europa}

\begin{tabular}{|l|c|}
\hline Costa de Marfil & $64,2 \%$ \\
\hline Costa Rica & $25,3 \%$ \\
\hline Ghana & $4,6 \%$ \\
\hline Camerún & $2,2 \%$ \\
\hline África Del Sur & $1,4 \%$ \\
\hline República Dominicana & $0,8 \%$ \\
\hline Tailandia & $0,4 \%$ \\
\hline Ecuador & $0,2 \%$ \\
\hline otros países & $0,9 \%$ \\
\hline
\end{tabular}

Fuente: Consejo Nacional de Producción (CNP, 2010).
Costa Rica es líder mundial en la exportación de piña fresca, en conjunto con Costa de Marfil, Filipinas (no aparece en la Tabla 1 por no ser Europa su principal mercado de piña fresca) y Francia. Por su parte, los entre los principales importadores de piña fresca son Francia, Italia, Alemania y el Reino Unido (el 90\% de la piña consumida en este país es de origen costarricense, (Ministerio de Relaciones Exteriores, Comercio e Integración de Ecuador, 2011), con un crecimiento anual de importaciones de 6,7\% 5,6\% 5,7\% y 4.4\% respectivamente (Inteligencia de mercados).

En la tabla 2 se puede apreciar cual fue la demanda de piña fresca de los principales consumidores de esta fruta.

TABLA 2

Principales países importadores de piña fresca

\begin{tabular}{|l|c|}
\hline \multicolumn{1}{|c|}{ País } & $\begin{array}{c}\text { Importación } \\
\text { en toneladas métricas }\end{array}$ \\
\hline Estados Unidos & 696820 \\
\hline Bélgica & 292499 \\
\hline Países Bajos & 200026 \\
\hline Alemania & 167416 \\
\hline Japón & 165794 \\
\hline Italia & 142168 \\
\hline Reino Unido & 116730 \\
\hline España & 113182 \\
\hline Canadá & 102064 \\
\hline Francia & 96488 \\
\hline
\end{tabular}

Fuente: CNP (2010)

Para el año 2008 y hasta el 2010 Costa Rica ocupa el cuarto lugar como productor mundial de piña de mesa. Siendo su fruta la de mayor demanda en los Estados Unidos de América y en Europa, con una participación del 12\% del mercado mundial; como se aprecia en el gráfico 1. 
GRÁFICO 1

\section{Principales países productores de piña, en toneladas métricas}

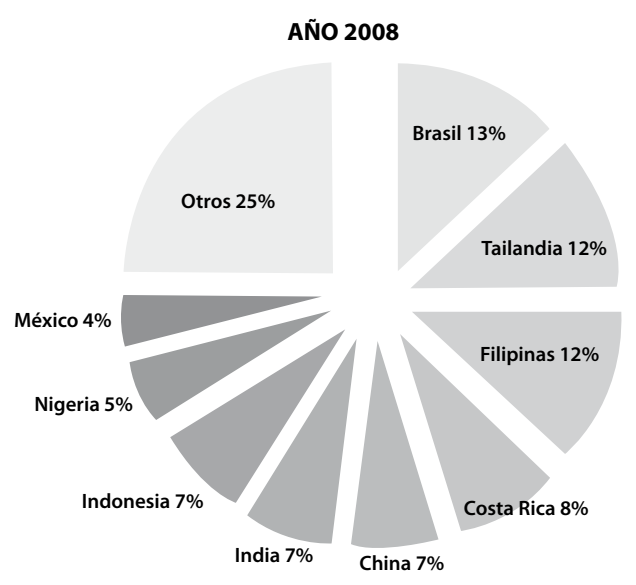

Fuente: FA0 (2004).

El $70 \%$ de la piña producida en el mundo es consumida como fruta fresca en el país que la produce.

Tailandia, Filipinas, Brasil y China son los principales productores de piña con cerca del $44 \%$ de la producción mundial (CNP, 2010). En tanto, Costa Rica está ligada a Indonesia, México, la India y otros países que producen el restante porcentaje de la piña mundial.

En cuanto a la producción de piña, y en comparación con los demás países productores, Costa Rica tiene una alta demanda de piña fresca, con predominio de la Unión Europea y de los Estados Unidos.

El crecimiento del cultivo en Costa Rica ha tenido un aumento sostenido desde 1980, fecha en que se inició la agroindustria de la piña.

El mercado de la piña fresca muestra un aumento en la demanda y una estabilidad en los precios. Costa Rica es el cuarto país a nivel mundial en exportaciones en fresco según el volumen de producción, pues exporta el $67,9 \%$ de su producción de fruta en dicho estado (FAO, 2004 y 2009 y CNP, 2010).

Doce naciones concentran el $90 \%$ de la demanda mundial de piña fresca, siendo Estados Unidos el mercado con mayor participación, ade- más de Francia, Japón, Bélgica, Italia, Alemania, Canadá, España, Reino Unido, Corea, Países Bajos y Singapur (Coveca, 2009). Para el año 2004, según la $\mathrm{FAO}^{3}$ (2004 y 2009) CNP (2010), Costa Rica ocupa el séptimo lugar en producción de piña en el mundo equivalente a $725224 \mathrm{tm}$.

En estos últimos años Costa Rica ha empezado a incursionar en el mercado de venta de jugo de piña, con una participación del mercado internacional del 5,6\% y con un crecimiento del $11 \%$. El mayor consumidor de jugo es los EEUU, con un $89 \%$ de la demanda del mercado mundial (Inteligencia de mercados, s.f).

Algo muy importante para la piña costarricense, es el nicho de la piña orgánica, donde Dinamarca se destaca, después de Francia, como el mayor consumidor de piña orgánica fresca en Europa, siendo Costa Rica uno de sus proveedores.

No todos los países han podido incursionar en el cultivo de piña orgánica, y Costa Rica tiene, en ese sentido, una ventaja en este tipo de producción, pues cuenta con los mayores importadores de piña fresca orgánica y se estima que la demanda continuará creciendo un $20 \%$ anual. El último trimestre de 2002 fue introducida al mercado la certificación Comercio Justo para frutas tropicales, con lo cual se crea una nueva categoría de "piña sostenible" que ha crecido rápidamente. El volumen del mercado de piña Comercio Justo se estima en 1,000 toneladas anuales para 2003. Actualmente, la totalidad de la producción es destinada a los mercados de Suiza, Dinamarca, Alemania e Inglaterra. Los países productores de piña "Comercio Justo" son Costa Rica y Ghana (Centeno, 2003).

En los últimos años el cultivo de piña orgánica y piña fresca ha tenido un incremento en la producción debido al aumento en la demanda a nivel internacional.

Los mercados de exportación de la piña costarricense son muy diversificados, siendo los princi-

3. Food and Agriculture Organization of the United Nations. 
pales compradores los EEUU y la UE. En el caso de Europa, Costa Rica exporta aproximadamente el 38\% del total consumido, es decir, unas 372 mil toneladas métricas, lo cual convierte al país en el mayor suplidor para el mercado europeo, arriba de los países asociados a la Unión Europea que exportan el 36\% del total.

Para el período Enero-Diciembre 2008 se exportaron $1444458 \mathrm{tm}$, por un valor FOB de $\$ 566,8$ millones, el precio promedio por kilogramo de piña costarricense exportada fue de $\$ 0,39 / \mathrm{kg}$. Los principales países donde se exportó la piña nacional fueron: EEUU, con una participación del 43\%; Bélgica, con el 19\%; Italia con una participación de compra del 10\%; y Holanda con el $8 \%$.

El precio de la piña costarricense es muy competitivo, hecho que es estratégico en el aumento de demanda del producto, no solo por su precio, sino por calidad. Aunado a ello, es importante destacar que la tendencia en la demanda de la piña costarricense no ha dejado de crecer.

Para el año 2010 se previó un aumento en la producción y exportación de piña, así mismo se espera que en 2011 la demanda de piña costarricense aumente, tanto en fruta fresca, fruta orgánica como en el nuevo producto: el jugo de piña. Además, se espera este mismo comportamiento con nuevas variedades que se están desarrollando en Pococí, con una piña que tiene sabor a manzana. En los primeros 10 meses del año 2010, Costa Rica colocó piña por un valor de $\$ 478$ millones a unos 30 países diferentes, según la Promotora del Comercio Exterior (PROCOMER).

La tendencia del consumo de frutas tropicales ha venido en claro aumento y la piña no es la excepción (EUROSTAT), sobre todo si se toman en consideración los datos históricos del comportamiento del mercado de la piña, el cual se mantiene en franco desarrollo. Esto es un indicador para inferir que en 2011 la demanda de piña a nivel global está en aumento, y Costa Rica, con su participación histórica de mercado y con la incursión de nuevos productos como el jugo de piña, experimentará, muy probablemente, un incremento en la demanda de dicho producto, aspecto que se suma al precio y calidad altamente competitivos que se exige en los mercados internacionales y que nuestro país cumple a cabalidad.

En cuanto a la demanda de la piña, tanto en fresco como industrializada, se aprecia que Costa Rica tiene por delante un futuro prometedor en términos de la producción y colocación de jugo de piña, ya sea procesada o simplemente empacada.

El aumento de la demanda tiene su asidero en los nuevos mercados de Turquía e Iraq. Esa combinación de factores, sumado a la oportunidad que significan las nuevas ventas en el mercado europeo, permitirán el repunte del sector para este año, según las estimaciones de CANAPEP.

En ese contexto, las nuevas estrategias de Costa Rica se apoyan en el desarrollo de diferentes variedades de piña, ya que las que habían desarrollado anteriormente, están siendo explotadas en todos los países del mundo. Todo esto hace que el precio de la piña Sweet o dorada, empiece a decaer. Sin embargo, con el desarrollo de las nuevas variedades como la de sabor a manzana, Costa Rica está tratando de asegurarse la expansión del mercado a futuro, así como nuevos mercados, nuevas variedades, nuevos productos y precios muy competitivos con la calidad exigida en el mundo.

Dentro de las tendencias para seguir haciendo negocios a futuro con la piña, está el desarrollo continuo de nuevas variedades para el mercado de fruta fresca como lo es la piña con sabor a manzana que se ha desarrollado en Pococí, además de productos como el jugo simple o procesado, las hojuelas deshidratadas, los pulverizados, las barras granulares y las salsas de piña. 


\section{Modelo de valoración de la gestión productiva de la piña}

El modelo que se presenta contribuye a reunir la información necesaria para determinar la eficiencia, eficacia y control de la producción de la piña hasta su venta. Con ello se da una herramienta, por medio de indicadores, que le permite al interesado saber sobre su rendimiento y su manejo, para poder facilitar, entre uno de sus beneficios, la certificación fitosanitaria del producto a fin de poder ingresar en este caso a Europa, que consume el $40 \%$ de la producción mundial de piña.

Hoy en día los países más ricos del mundo, con los mayores estándares de vida, han venido desarrollando una conciencia colectiva muy arraigada en los temas ecológicos y ambientales, por lo que son muy escrupulosos a la hora de seleccionar los alimentos que ingieren. Esto hace que tengan una preferencia muy marcada por los productos elaborados y cosechados con los mayores estándares, destacando los cosechados orgánicamente y en el marco de producciones sostenibles.

Antes de presentar el Modelo Valorativo de la Gestión Productiva de la Piña para certificación de las fincas y empacadoras, se detallará cómo es el manejo de plagas, así como las variables que determinan su transibilidad, y la equivalencia de los operadores de la piña sobre su inocuidad de la piña. Tambien así con lo relacionado a la higiene de esta y su contenido de límites máximos de residuos de carbaril, deltametrin, endosulfan, fenitrotion, metidation y oxami.

Tambien se expondrá el proceso del manejo de la piña desde la siembra, hasta la exportación, datos que se desprenden de las entrevistas a profundidad y semiestructuradas realizadas a Raúl López Álvarez de la empresa Productora y exportadora FRUVER, S. A., la cual exporta piña a Europa.

\section{Proceso de producción de piña por la empresa FRUVER}

Para cultivar un terreno con piña, la preparación supone un período no menor de tres meses antes de plantar, a fin de lograr la descomposición de los residuos vegetales. Se necesitan alrededor de entre 12 y 14 meses para que la planta empiece su cosecha.

El cultivo de piña requiere de suelos de buen drenaje, es decir, un suelo permeable y franco limoso. Además se necesita un clima húmedo y una temperatura que oscile entre los $25^{\circ} \mathrm{C}$ y los $32^{\circ} \mathrm{C}$. La piña es un cultivo perenne y corresponde a la categoría de las herbáceas.

\section{Variedad de piña sembrada}

La piña que se cultiva en la empresa FRUVER, es la conocida en Costa Rica como la piña amarilla o dorada, también llamada MD2, es un cultivo de piña híbrida derivado de la piña Cayena. La planta es de rápido crecimiento, lo cual redunda en un ciclo de producción más corto; además, los rendimientos de producción y de tamaño de la fruta son altos y es una fruta muy dulce y jugosa, aunque más susceptible al daño mecánico que la Champaka. Su densidad óptima para el cultivo se ubica entre 65000 y 75000 plantas por hectárea.

Actualmente la piña dorada es la de mayor auge y preferencia en el mercado internacional por sus atributos sensoriales. Esto hace que logre mayores precios que otras variedades.

Para el cultivo de la piña en los terrenos de cultivo se deben realizar las siguientes acciones a fin de tener un producto de máxima calidad que le permita al productor colocar al mejor precio la piña cultivada en el mercado internacional, en este caso en Europa.

Las acciones que se deben realizar para lograr un producto de máxima calidad son: 
1. Preparación de suelo: la preparación de la tierra se realiza con rastra y subsolador. Es importante que el terreno quede libre de malezas y bien afinado.

2. Encamado: la formación de camas se hace mecánicamente mediante el uso de una encamadora.

3. Drenaje: esta medida se toma para eliminar erosión por parte del agua y prevenir estancamiento de agua que va afectar el cultivo

4. Siembra: este cultivo se puede sembrar todo el año.

5. Selección de semilla: debe seleccionarse la semilla por tamaño y tipo, para uniformizar cada área de cultivo (La semilla la dará la misma planta después del proceso de cosecha).

Las acciones relacionadas con el manejo del cultivo son:

1. Fertilización: el Nitrógeno y el Potasio son los nutrimentos más importantes para la piña; el primero influye sobre el rendimiento y el segundo sobre la calidad de la fruta, principalmente. En los primeros estados de desarrollo se recomienda la aplicación de fósforo para contribuir a un buen desarrollo radical.

2. Control de malezas: la finalidad del control de las malezas es evitar la competencia entre estas y la piña, principalmente, y entre agua y nutrientes.

3. Control de plagas y enfermedades: se realiza por medio de insecticidas y tratamientos. Para mayor comodidad y rapidez en la aplicación se utiliza un spray boom y sus correspondientes nodrizas para la recarga del mismo.

Cuando la planta ha alcanzado un peso mínimo de 3000 gramos, se debe realizar una inducción floral por medio de gas etileno, carbón activado y agua para garantizar una floración homogénea y precisa.

Es preciso conocer el período que transcurre entre la inducción de la floración y la cosecha. A partir de 150 días (5 meses) de realizada la inducción floral, se debe estar alerta y hacer inspecciones a fin de observar el estado de desarrollo, el tamaño y el grado de madurez alcanzado por la fruta. Para darle el color deseado se hace una aplicación de un producto días antes de la cosecha para afinar la coloración de los frutos.

La cosecha se realiza en forma manual y posteriormente se utiliza una cosechadora para llevarla hasta la carreta. Para el transporte a la empacadora, se utilizan cajas plásticas que impedirán el maltrato de la fruta.

Al momento de cosechar se debe tomar en cuenta el color del fruto o los grados brix (12\% y $13 \%$ ), así como la traslucidez.

Se debe de evitar el calentamiento de la fruta en días soleados tanto en el campo como durante el transporte.

\section{Manejo de la piña despues de cosechada}

Una vez realizada la recolección o cosecha de la piña, se lleva al plantel para su proceso. La descarga de la fruta se hace manualmente sobre las pilas con agua (se coloca fruta por fruta dentro de los tanques). En esos tanques, la fruta se lava y desinfecta. Generalmente se emplea agua clorinada. En las pilas de lavado se desechan las frutas que se hunden y el resto sale por un elevador en uno de los extremos del tanque que lleva la fruta a las bandas de selección. El proceso de selección se hace manualmente y consiste en la eliminación del producto que no cumple con los requerimientos de calidad. Además se realiza una serie de procedimientos con la piña para poderla hacer llegar al consumidor, los cuales son los siguientes: 
1. Encerado y aplicación de fungicidas: el encerado en la piña se hace solamente a la fruta y no a la corona. Se utiliza un sistema de cascada. Se hace con el fin de mejorar la apariencia de la fruta, protegerla y disminuir problemas relacionados con el envejecimiento normal de los frutos. Este proceso puede retardar el cambio de color externo de la fruta.

2. Clasificación y empaque: tiene por objeto separar las frutas según su color y según su tamaño. Este proceso se hace conjuntamente con el proceso de empaque. Se utilizan cajas para un peso neto de piña entre 11 y $12 \mathrm{Kg}$.

Los tamaños de los frutos de piña, dados en función de la cantidad de frutos que se empacan en una caja de $12 \mathrm{Kg}$., varían del No. 5 al No. 10. La fruta se coloca en cajas de cartón corrugado de una sola capa. A cada fruta se le coloca una etiqueta informativa.

Las cajas llenas se pesan para comprobar el peso, el cual debe ser ligeramente mayor de lo que indica la caja, para compensar la pérdida de humedad del producto durante el transporte.

3. Paletizado: la formación de paletas o tarimas permite agilizar la manipulación del producto empacado. Las cajas se colocan sobre tarimas de madera de 1,0 × 1,2 m. y cada tarima lleva una cantidad de 75 a 80 cajas, para un total por contenedor de 1500 a 1560.

4. Enfriamiento: las condiciones óptimas para el almacenamiento y transporte de la piña son de $7.5^{\circ} \mathrm{C}$ con un $85 \%$ a $90 \%$ de humedad relativa. Es importante reducir la temperatura de la fruta tan pronto como sea posible para disminuir la velocidad del deterioro del producto y extender su vida comercial.

La piña se enfría primero en una cámara de aire forzado para bajar la temperatura de esta de aproximadamente $30^{\circ} \mathrm{C}$ que viene del campo a $7.5^{\circ} \mathrm{C}$ que se requiere. Posteriormente se pasa a unas cámaras de mantenimiento en espera de ser cargadas dentro del contenedor que irá con dirección al puerto, por medio del muelle de carga sus correspondientes sellos a la puerta del equipo refrigerado para evitar rompimiento de la cadena de frío.

Antes de introducir las paletas, se debe revisar el contenedor (higiene, libre de productos químicos, residuos de materia orgánica, estado de las paredes y puertas, registro de lavado y desinfección, registro de viajes anteriores para verificar que sea aceptable para el transporte de alimentos y otros), comprobar la buena operación de la unidad de refrigeración, el termostato, el ajuste de los cambios de aire y los controles de temperatura; el contenedor se debe enfriar hasta la temperatura de la cámara.

5. Transporte: el transporte de la piña a terceros mercados se hace por vía marítima en la mayor parte de los casos. El costo del transporte aéreo es elevado y la disponibilidad limitada.

El tiempo de transporte varía según el mercado de destino. Hacia los EE.UU. el viaje tarda alrededor de 5 a 7 días y para Europa de 12 a 20 días.

\section{Modelo y evaluación}

El modelo que se propone se considera medular en una empresa agroindustrial dedicada a la explotación de piña tradicional u orgánica, pues está orientado a que en su proceso de siembra, manejo y transporte, el producto no se contamine, ni contenga químicos y pesticidas en el caso de la piña orgánica, y busca que se utilice la menor cantidad posible de agroquimicos en la producción de piña tradicional (no orgánica).

Se propone un modelo que visualice las finanzas (son muy simples), las estrategias de mercadeo 
y comercialización, que normalmente están en manos de grandes transnacionales, las cuales cuentan con los canales adecuados para penetrar un mercado tan exigente y selecto como el de los paises desarrollados, máxime porque muchas de estas transnacionales tienen todo su proceso integrado, pero también acopian fruta a pequeños productores nacionales. De ahí que estos deban enfocarse en la utilización óptima de los insumos, en el manejo ideal del proceso y en la calidad del producto.

En virtud de lo anterior, se expone un modelo global para una empresa, con el fin de aclarar y visualizar las interaciones que se dan en esta industria. Se procede a exponer el modelo propuesto para esta investigación, el cual se aprecia mediante el siguiente flujograma (Figura 1).
Este modelo está dividido en cuatro partes o fases; cada una tendrá un valor específico. Así, la fase de insumos tendrá un peso relativo de 30\%, la fase del proceso un 20\%, la fase del producto un 30\% y por último la fase del cliente un 20\%. Se le da un peso más bajo a clientes, porque si se cumple bien con las tres primeras partes, hay gran certeza de poder colocar el producto en el mercado meta. Una calificación de por lo menos aceptable de todo el proceso pondera a la empresa como una organización competitiva dentro del mercado internacional, siendo aceptable una calificación no menor al 70\% de la suma de todos los indicadores dentro de cada fase.

En cada fase o parte del modelo presentado se valora la eficacia, efectividad, proceso e impacto.

FIGURA 1

\section{Resumen del Modelo de gestión de la piña}

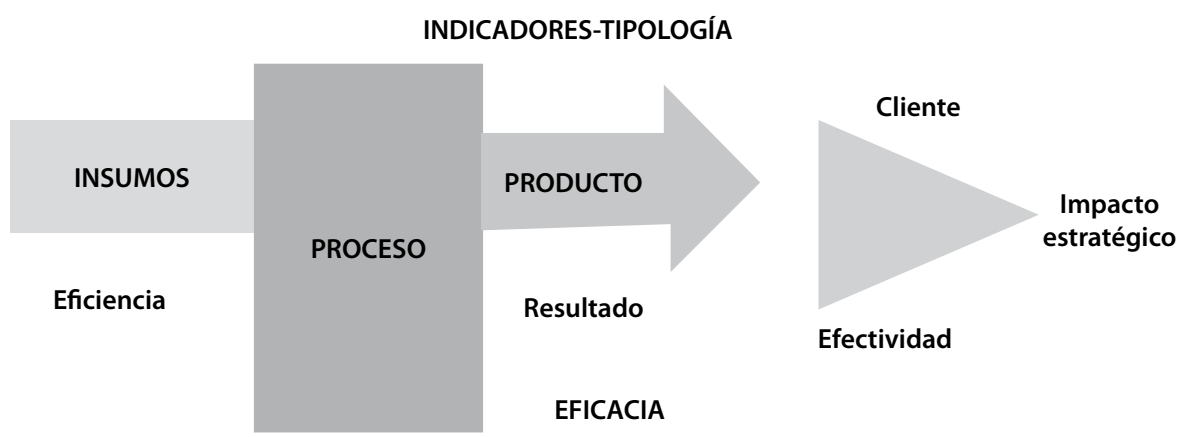

\begin{tabular}{|c|c|c|c|}
\hline Proceso & Eficacia & Efectividad & Impacto \\
\hline Recursos & Calidad & Satisfacción & Marca \\
\hline Materiales & Oportunidad & Participaciòn & Credibilidad \\
\hline Métodos & Confiabilidad & Productividad & Valor agregado \\
\hline Mano de obra & & & \begin{tabular}{c} 
Reconocimiento \\
\hline Tiempo
\end{tabular} \\
\hline
\end{tabular}

Fuente: elaboración propia. 
TABLA 3

Fases a evaluar del proceso agroindustrial de la piña

\begin{tabular}{|l|l|l|l|}
\hline $\begin{array}{l}\text { Fases: } \\
\text { 1. Insumo } \\
\text { 2. Proceso } \\
\text { 3. Producto } \\
\text { 4. Cliente }\end{array}$ & \multicolumn{1}{|c|}{ Eficacia } & Efectividad & \multicolumn{1}{|c|}{ Impacto } \\
\hline Recursos & Calidad & Satisfacción & Marca \\
\hline Materiales & Oportunidad & Participación & Credibilidad \\
\hline Métodos & Confiabilidad & Productividad & $\begin{array}{l}\text { Valor } \\
\text { agregado }\end{array}$ \\
\hline $\begin{array}{l}\text { Mano de } \\
\text { obra/talento } \\
\text { humano }\end{array}$ & & & Reconocimiento \\
\hline Tiempo & & & $\begin{array}{l}\text { Contribución } \\
\text { estratégica }\end{array}$ \\
\hline
\end{tabular}

Fuente: elaboración propia.

Los indicadores se cuantificarán según la tabla 4 , y se resumen como se presenta en la tabla 5. En vista de que el mercado desarrollado es muy exigente, solo la calificación aceptable como mínima, sería la calificación adecuada para poder exportar el producto.

TABLA 4

\section{Calificaciones de los indicadores}

\begin{tabular}{|l|c|c|c|}
\hline Disposiciones & Abreviación & Puntos & Porcentaje \\
\hline Correcta & C & 10 & $85-100$ \\
\hline Aceptable & A & 8 & $70-84$ \\
\hline Regular & R & 6 & $55-69$ \\
\hline Mala & M & 4 & $0-54$ \\
\hline
\end{tabular}

Fuente: elaboración propia.

TABLA 5

Resumen de resultados

\begin{tabular}{|l|l|}
\hline \multicolumn{1}{|c|}{ Fase } & PRR $^{4}$ \\
\hline Insumos & \\
\hline Proceso & \\
\hline Producto & \\
\hline Clientes & \\
\hline Total obtenido & \\
\hline
\end{tabular}

Fuente: elaboración propia.
El desarrollo del modelo se presenta a continuación, donde se desglosan las tablas en las cuales se registran los diferentes datos obtenidos en cada fase del modelo; luego se suman y se obtiene una calificación del producto final, lo cual permite visualizar dónde se está fallando y en qué etapa de la fase se debe mejorar, para tener mejores resultados, estos resultados se deben de poner en la cédula de análisis de insumo, proceso, producto y cliente que está la tabla 4.

\section{Forma de interpretación de los datos en las diferentes fases o etapas el modelo}

En las siguientes 4 tablas del modelo de gestión agroindustrial de la piña, se dan una serie de indicadores, los cuales se deben ir rellenando.

$H R=$ Indica la hoja de referencia en la cual se aplicó la evaluación que se plasma en el modelo.

$\mathrm{COI}=$ Calificación obtenida en letras de los diferentes instrumentos $u$ hojas que se emplearon para la evaluación cuantitativa, que se expresa de manera cualitativa: C: correcta, A: aceptable, R: regular, M: mala.

$\mathrm{CN}=$ es la cantidad obtenida de la evaluación cuantitativa en porcentajes y no en letras.

Peso \% = es el peso relativo de cada ítem a examinado, de cada fase.

VR $=$ Valor relativo. Es el resultado de CN * Peso \%.

$\mathrm{DI}$ = Es la proporción en que se ajusta el indicador.

$\mathrm{PR}=$ Peso relativo de cada índice en cada fase.

PR-Resultado $=$ Es el resultado de la multiplicación de Peso* el peso relativo de cada fase. Con esto se obtiene un valor relativo de contribución al aporte de la evaluación de la gestión productiva.

PR-Ideal = es el porcentaje máximo obtenido si cada indicador se cumpliese en el 100\%.

CGA = Calificación general de la gestión por área. Partiendo del hecho de que a cada área se 
le asignó un determinado porcentaje (entre $20 \%$ y $30 \%$ ), este resultado se obtiene de dividir la sumatoria de la fase correspondiente (PRR) sobre la sumatoria de PR-Ideal x 100, lo que da un porcentaje, que hay que mirar en la tabla 6 para definir su calificación numérica y cualitativa, in- dicando con ello la calificación de la gestión de la empresa en dicha área.

Evaluación integral de gestión. La sumatoria de PR-Real, en las cuatro áreas, indica la calificación numérica de la gestión en una escala de 1 a 100.

TABLA 6

Fase de Insumos

\begin{tabular}{|c|c|c|c|c|c|c|c|c|}
\hline \multicolumn{9}{|c|}{ FASE DE INSUMOS } \\
\hline \multirow{2}{*}{ Indicadores } & \multirow{2}{*}{$\mathrm{HR}$} & \multirow{2}{*}{$\mathrm{cOl}$} & \multirow{2}{*}{$\mathrm{CN}$} & \multirow{2}{*}{ Peso \% } & \multirow{2}{*}{ VR } & \multirow{2}{*}{ DI\% } & \multicolumn{2}{|c|}{ PR } \\
\hline & & & & & & & Resultado & Ideal \\
\hline Indice de calidad & & & & 20 & & & & 6 \\
\hline Indice de confiabilidad & & & & 20 & & & & 6 \\
\hline Índice de satisfacción & & & & 20 & & & & 6 \\
\hline Índice de efectividad de semilla & & & & 35 & & & & 10,5 \\
\hline Tiempo perdido por incumplimiento de proveedores & & & & 5 & & & & 1,5 \\
\hline Subtotal de la fase & & & & 100 & & & & 30 \\
\hline
\end{tabular}

Fuente: elaboración propia.

TABLA 7

Fase de Procesos

\begin{tabular}{|c|c|c|c|c|c|c|c|c|}
\hline \multicolumn{9}{|c|}{ FASE DE PROCESO } \\
\hline \multirow{2}{*}{ Indicadores } & \multirow{2}{*}{ HR } & \multirow{2}{*}{$\mathrm{COI}$} & \multirow{2}{*}{$\mathrm{CN}$} & \multirow{2}{*}{ Peso \% } & \multirow{2}{*}{ VR } & \multirow{2}{*}{$\mathrm{DI} \%$} & \multicolumn{2}{|c|}{ PR } \\
\hline & & & & & & & Resultado & Ideal \\
\hline Recursos & & & & 20 & & & & 4 \\
\hline Métodos & & & & 15 & & & & 3 \\
\hline Mano de obra calificada & & & & 15 & & & & 3 \\
\hline Confiabilidad & & & & 20 & & & & 4 \\
\hline Productividad & & & & 30 & & & & 6 \\
\hline \multicolumn{4}{|c|}{ Subtotal de la fase } & 100 & & & & 20 \\
\hline
\end{tabular}

Fuente: elaboración propia.

TABLA 8

Fase de Producto

\begin{tabular}{|c|c|c|c|c|c|c|c|c|}
\hline \multicolumn{9}{|c|}{ FASE DE PRODUCTO } \\
\hline \multirow{2}{*}{ Indicadores } & \multirow{2}{*}{ HR } & \multirow{2}{*}{$\mathrm{COI}$} & \multirow{2}{*}{$\mathrm{CN}$} & \multirow{2}{*}{ Peso \% } & \multirow{2}{*}{ VR } & \multirow{2}{*}{ DI \% } & \multicolumn{2}{|c|}{ PR } \\
\hline & & & & & & & Resultado & Ideal \\
\hline Satisfacción & & & & 20 & & & & 6 \\
\hline Participación del mercado de exportación & & & & 15 & & & & 4,5 \\
\hline Productividad & & & & 30 & & & & 9 \\
\hline Rechazos & & & & 20 & & & & 6 \\
\hline Calidad óptima para Exportación & & & & 15 & & & & 4,5 \\
\hline \multicolumn{4}{|c|}{ Subtotal de la fase } & 100 & & & & 30 \\
\hline
\end{tabular}

Fuente: elaboración propia. 
TABLA 9

Fase Cliente

FASE CLIENTE

\begin{tabular}{|c|c|c|c|c|c|c|c|c|}
\hline \multirow{2}{*}{ Indicadores } & \multirow{2}{*}{ HR } & \multirow{2}{*}{$\mathrm{COI}$} & \multirow{2}{*}{$\mathrm{CN}$} & \multirow{2}{*}{ Peso \% } & \multirow{2}{*}{ VR } & \multirow{2}{*}{ DI \% } & \multicolumn{2}{|c|}{ PR } \\
\hline & & & & & & & Resultado & Ideal \\
\hline Marca & & & & 10 & & & & 2 \\
\hline Credibilidad empresa y país & & & & 25 & & & & 5 \\
\hline Valor agregado & & & & 25 & & & & 5 \\
\hline Reconocimiento de origen y calidad orgánica & & & & 10 & & & & 2 \\
\hline Contribución estratégica(distribuidor) & & & & 30 & & & & 6 \\
\hline \multicolumn{4}{|l|}{ Subtotal de la fase } & 100 & & & & 20 \\
\hline
\end{tabular}

Fuente: elaboración propia.

Para una mejor comprensión de estas fases en el anexo se da una explicación de cómo se debe de llenar e interpretar las tablas 6, 7, 8 y 9.

\section{CONCLUSIONES}

Con el "modelo de valoración de la gestión productiva de la piña" se realiza una contribución principalmente a las MiPymes agroindustriales de la piña, que también se puede utilizar en cualquier otro cultivo agroindutrial. Uno de los productos resultantes de este modelo es la esquematización de toda la actividad productiva de la piña desde su siembre hasta la venta, con el cual se puede evidenciar dónde se es muy competitivo y dónde se debe mejorar; como por ejemplo en la técnica de siembra, recolección, o tal vez en el procesamiento de la fruta y hasta cuantificar la aceptación del producto en el mercado final.

Además se evidencian indicadores ecenciales para una asertada admnistración de la actividad que contribuya a ser más competitivo en el mercado internacional por rendimiento de mano de obra y rendimineto de terrenos, una de las ventajas del país es el altísimo grado de investigación que se da y se tiene sobre la piña, lo que lo ha mantenido a la vanguardia mundial, pero esta ventaja muy rápido se pierde cuando los países competidores adquieren las plantas desarrolladas en Costa Rica, lo cual pone al país y a sus produc- tores en desaventaja por las altas cargas sociales que se manejan en nuestro territorio.

Con el modelo propuesto se pretende contribuir al mejoramiento de la actvidad agroindustrial de la piña, brindando un instrumento que contribuya a la evaluación del proceso productivo de la piña tradicional y orgánica, desde la preparación de los terrenos, la cosecha, el procesamiento, así como la distribución para un mercado selecto y exigente con los productos agrícolas.

De esta forma, el administrador de la empresa agroindustrial podrá brindar documentación cualitativa y cuantitativa sobre el proceso de la producción y la penetración del producto en los mercados internacionales. Además, el instrumento permitirá que la producción tienda a mejorar sus rendimientos financieros, pues pondrá en evidencia los puntos débiles del proceso en general.

También es un instrumento que contribuye a tener un historial de las diferentes fases del cultivo, así como los diferentes agroquímicos y sus cantidades utlizadas, además de la calidad obtenida de fruta, para que las visitas que periódicamente realiza la Unión Europea para certificar las fincas de produción y empacadoras de piña de Costa Rica, así como la certificación fotosanitaria de la piña, se puedan realizar con resultados óptimos; al tiempo que le permitirá a las empresas posicionar aún mejor su producción, tanto en el mercado de la comunidad Europea, como en el resto del mundo. 


\section{REFERENCIAS}

Acuña, G. (2006). Producción de piña en caribe y pacifico sur de Costa Rica. Revista Ambientito, 158. Recuperado de http://www.ambientico.una.ac.cr/158pdf

C.N.P. (2010) Análisis de mercado de la piña. Boletín Nº 1, año 15. Recuperado de http://cep.unep.org/repcar/ Bol_PINA1\%20junio\%202010.pdf

Centeno, P. (2003). CIMS for Organic Monitor. Recuperado de http://www.planetaorganico.com.br

Collins, J. L. (1960). The pineapple. London: Leonard Hill.

Collins, J.L. (1949). History, taxonomy and culture of the pineapple. Economic Botany, 3(4), 335.

Comisión Veracruzana de Comercialización Agropecuaria (Coveca). (2009). Monografía de la piña. México: Gobierno del Estado de Veracruz. Recuperado en http:// portal.veracruz.gob.mx/pls/portal/docs/PAGE/COVECAINICIO/IMAGENES/ARCHIVOSPDF/ARCHIVOSDIFUSION/MONOGRAFIA\%20PI\%D1A2010.PDF

Cultivo de piña con perspectivas para mercado internacional. (2010). Recuperado de http://agrytec.com/ agricola/index.php?option=com_content\&view = article\&id=278: cultivo-de-pina-con-perspectivaspara-mercado-internacional \&catid=47:articulostecnicos\&ltemid=34

De la Cruz, J. y García, H. FAO. Instituto Tecnológico de Veracruz Cap. XXXIII: Operaciones postcosecha. En http:// www.fao.org/inpho/content/compend/text/ch33s/ AE614s01.htm

FAO. (2004). Situación actual y perspectivas a mediano plazo para las frutas tropicales. En http://www.fao.org/es/ esc/common/ecg/218/es/Sit_web_s.pdf

FAO. (2004). En http://www.fao.org/livestock/agap/frg/ afris/espanol

FAO. (2009). The market for organic and fair-trade mangoes and pineapples. En http://www.fao.org/fileadmin/ templates/organicexports/docs/Market_Organic_FT_ Pineapple_Mango.pdf

Foodmarketexchange. (2007). En http://www.foodmarketexchange.com/datacenter/product/fruit/pineapple

Infoagro.com El mercado de las frutas tropicales en la unión europea. En http://www.infoagro.com/frutas/frutas_ tropicales/tropical_fruits.htm
Inteligencia de mercados. Perfil de Mercado, N 15. Ministerio de Agricultura y Desarrollo Rural, Colombia. ISSN 0124-1338. En http://www.dominicanaexporta.gov. do/Modulos/BibliotecaVirtual/Files/Perfil\%20de\%20 la\%20Pina.pdf

Llanos, M. 1998. Producción y comercio mundial de frutos tropicales. Revista Valencia Fruits No. 1881. Pag 1.

Piñeros prevén recuperar crecimiento en el 2010. En http:// wvw.nacion.com/In_ee/2009/diciembre/21/economia2201185.html

PNUD (4 de noviembre de 2010). hdr.undp.org (ed.): «"Informe sobre Desarrollo Humano 2010 Edición del Vigésimo Aniversario"» (en español) (PDF). Consultado el 04/11/10.

PROCOMER. (2010). Exportación de Piña, de Costa Rica. Recuperado de http://www.procomer.com/Espanol/ Estadisticas-04/seguimiento_sectorial/seguimiento\%20sectorial\%202010/seg_sect_set_10/Seg_Export_pina_set_10.pdf

Salazar, O. (s.f.) La producción de piña en Costa Rica. ASEROLA. Recuperado de http://www.aseprola.org/leer. php/145

Procomer. Ministerio de Comercio Exterior. (2007) Sector Piña. Recuperado de http://www.aacue.go.cr/comercio/sectoriales/presentaciones/Pi\%C3\%B1a.pdf

Proecuador. (2011). Boletín mensual de comercio exterior - Ministerio de Relaciones Exteriores, Comercio e Integración. Unidad de Inteligencia Comercial. AÑO 1 - NúMERO 4. Abril

Recibido: 30-05-2011

Aceptado: 04-11-2011

\section{Anexos}

Descripción de los índices del modelo

A continuación se presentan las tablas 10,11,12 y 13 en las que se explica cómo se deben de llenar las tablas 6, 7, 8 y 9 del Modelo de Gestión para la Piña. Aquí se detalla cómo se debe interpretar y cuantificar los diferentes indicadores que se consideran dentro de cada una de las 4 fases del modelo. 
TABLA 10

Descripción fase de insumos

\begin{tabular}{|c|c|c|}
\hline Indicador & Descripción & Forma de cálculo \\
\hline \multicolumn{3}{|c|}{ FASE DE INSUMOS } \\
\hline Índice de calidad & $\begin{array}{l}\text { Se cuantifican el cumplimiento de los } \\
\text { productos, con las normas de parámetros } \\
\text { de agricultura. }\end{array}$ & $\begin{array}{l}\text { Porcentaje del cumplimiento de las } \\
\text { regulaciones para agricultura }\end{array}$ \\
\hline \multirow{2}{*}{ Índice de confiabilidad } & \multirow{2}{*}{ Rendimiento de los insumos. } & Rendimiento obtenido del insumo $\times 100$ \\
\hline & & Rendimiento esperado \\
\hline Índice de satisfacción & $\begin{array}{l}\text { Rendimiento de frutos por planta } \\
\text { por hectárea por corte. }\end{array}$ & (Cantidad de fruta por hectárea por corte/70 000)*100 \\
\hline $\begin{array}{l}\text { Índice de efectividad de la } \\
\text { semilla y abonos }\end{array}$ & $\begin{array}{l}\text { Desarrollo de la planta; bajo, medio, } \\
\text { normal, sobresaliente. }\end{array}$ & $\begin{array}{l}\text { (Bajo }=30, \text { medio }=60, \text { normal }=90, \\
\text { sobresaliente }=100)^{*} 100\end{array}$ \\
\hline $\begin{array}{l}\text { Tiempo perdido por } \\
\text { incumplimientos }\end{array}$ & $\begin{array}{l}\text { Se cuantifica cuantas horas/hombre, } \\
\text { se pierden por incumplimiento de } \\
\text { los proveedores. }\end{array}$ & (Horas perdidas/horas laboradas)X 100. \\
\hline
\end{tabular}

Fuente: elaboración propia.

TABLA 11

Descripción fase de proceso

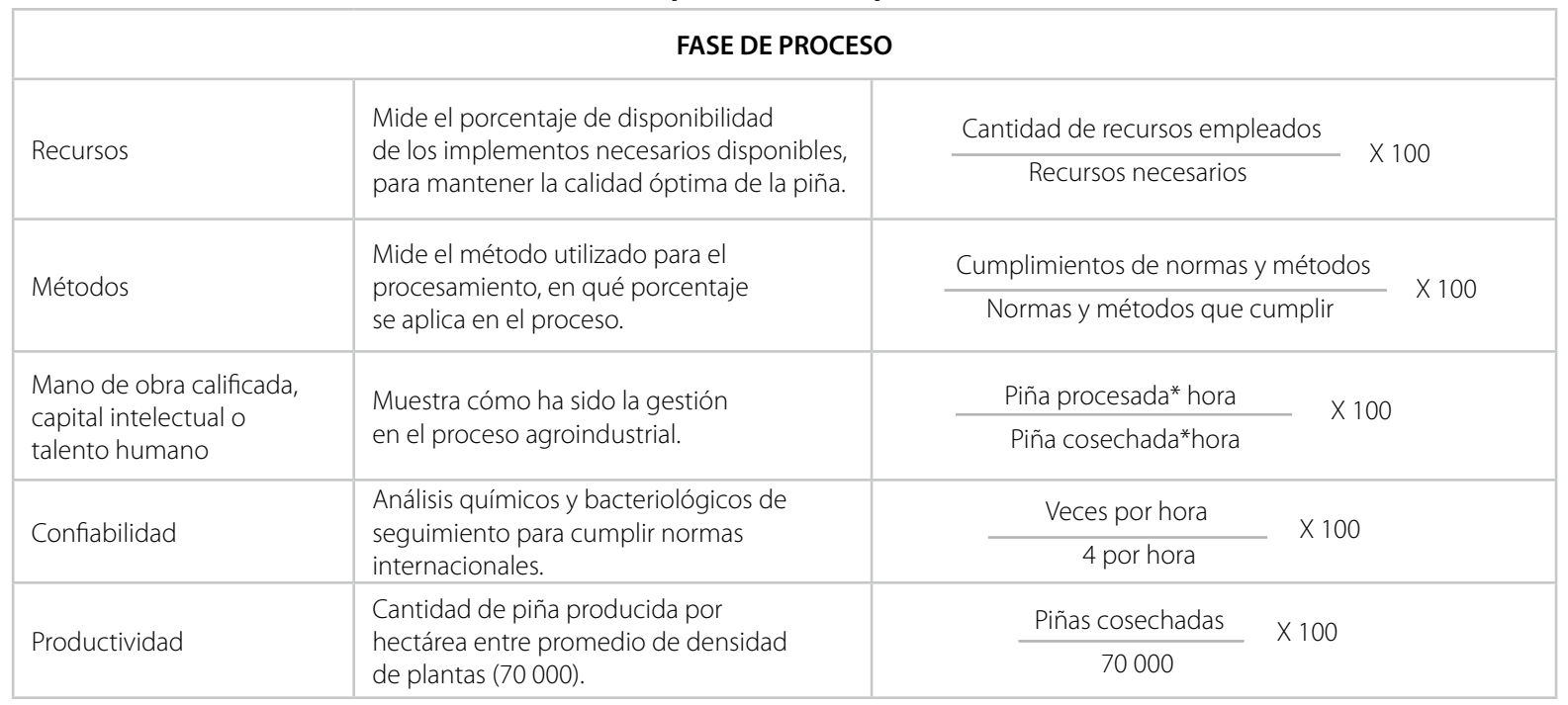

Fuente: elaboración propia. 
TABLA 12

\section{Descripción fase de producto}

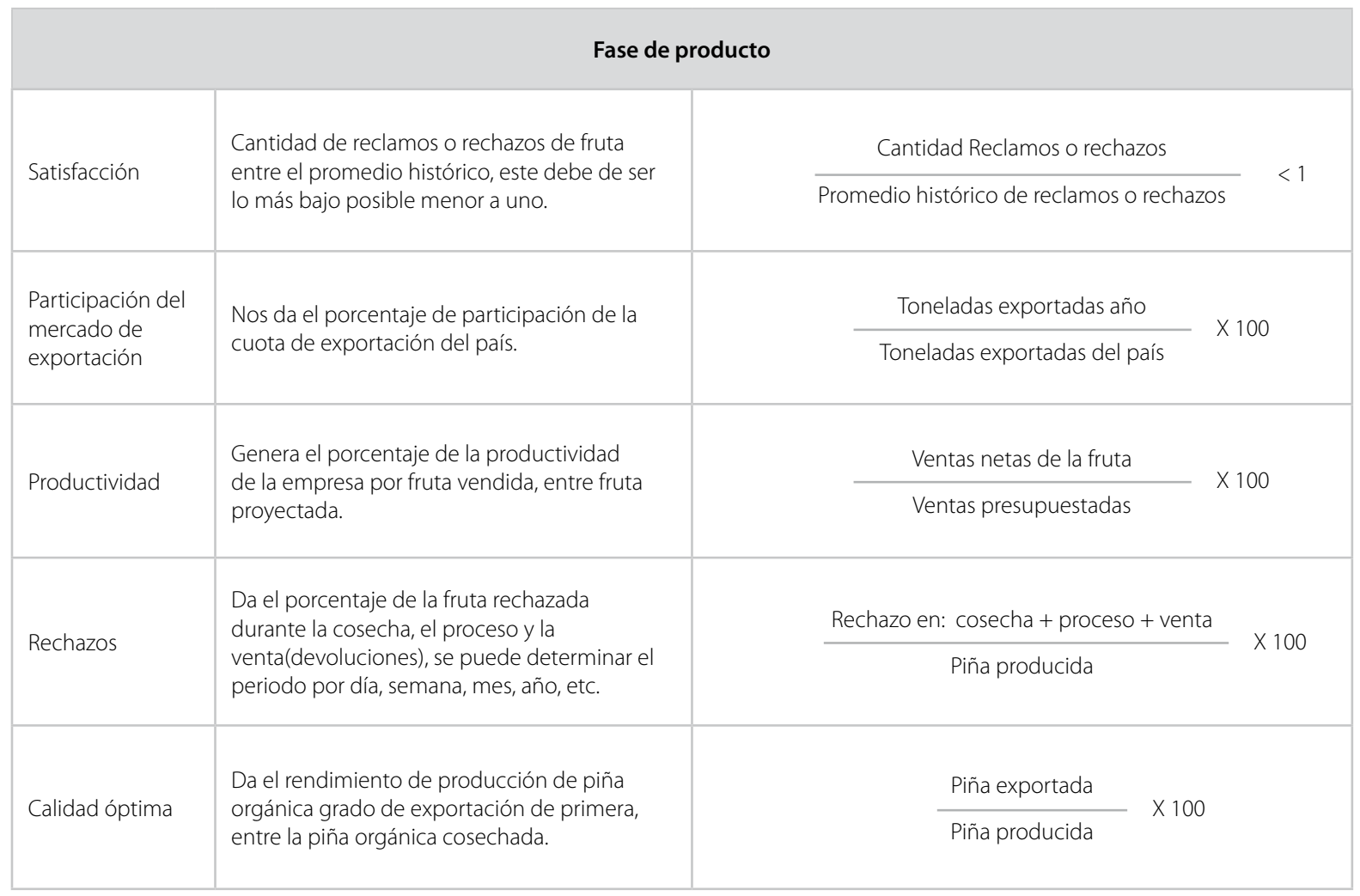

Fuente: elaboración propia. 
TABLA 13

Descripción fase cliente

\begin{tabular}{|c|c|c|}
\hline \multicolumn{3}{|c|}{ FASE CLIENTE } \\
\hline \multirow{2}{*}{ Marca } & \multirow{2}{*}{$\begin{array}{l}\text { Genera el nivel de penetración } \\
\text { de la marca (fruta), indica la } \\
\text { cobertura de distribución en } \\
\text { una zona, región o país. }\end{array}$} & Cantidad de negocios que comercializa la piña de la empresa \\
\hline & & Cantidad de negocios que comercializa la piña en región, país $x$ IUU \\
\hline \multirow{2}{*}{$\begin{array}{l}\text { Credibilidad } \\
\text { empresa y país }\end{array}$} & \multirow{2}{*}{$\begin{array}{l}\text { Es la recordación de manera } \\
\text { espontánea del producto, así } \\
\text { como del país de procedencia. }\end{array}$} & (\# menciones de la marca X 50) * (\# menciones del país X 50) \\
\hline & & Total de entrevistados \\
\hline \multirow{2}{*}{ Valor agregado } & \multirow{2}{*}{$\begin{array}{l}\text { Mide la calidad del producto, } \\
\text { así como la calidad de servicio, } \\
\text { ponderado entre precio } \\
\text { competitivo, entrega a tiempo } \\
\text { y completa, más posventa. }\end{array}$} & Calidad producto + calidad servicio \\
\hline & & Precio competitivo + entrega satisfactoria + posventa \\
\hline $\begin{array}{l}\text { Reconocimiento } \\
\text { de origen y calidad }\end{array}$ & $\begin{array}{l}\text { Este índice cuantifica las } \\
\text { certificaciones al día de } \\
\text { origen, procedencia, calidad } \\
\text { y certificaciones de los insumos } \\
\text { utilizados. Cada parámetro tiene } \\
\text { una valoración de } 1 \text {, si están al } \\
\text { día los documentos o de cero } \\
\text { sino están. }\end{array}$ & $($ parámetro a x 0,33) $+($ parámetro b x 0,33) $+($ parámetro $c \times 0,33)=1$ \\
\hline \multirow{2}{*}{$\begin{array}{l}\text { Contribución } \\
\text { estratégica } \\
\text { (distribuidor, } \\
\text { referenciamiento } \\
\text { competitivo global) }\end{array}$} & \multirow{2}{*}{$\begin{array}{l}\text { Mide el grado de } \\
\text { competitividad a nivel global } \\
\text { del distribuidor y del productor. }\end{array}$} & $\begin{array}{l}\text { Estándares de desempeño global } \\
(\text { empresa } \times, 50)+(\text { distribuidora } \times 0,50)\end{array}$ \\
\hline & & $\begin{array}{l}\text { Estándares de la mejores de la clase } \\
(\text { empresa } \times, 50)+(\text { distribuidora } \times 0,50)\end{array}$ \\
\hline
\end{tabular}

Fuente: elaboración propia.

1. PRR = se genera de cada cédula de análisis de insumo, proceso, producto y cliente.

1442 (2), Julio - Diciembre, 2011 